\title{
Síndrome de Kernohan. Descripción de caso y revisión de la literatura
}

\section{Kernohan Notch Syndrome. A case report and literature review}

Osvaldo M. San Martín-García* y Julio C. Soto-Barraza

Servicio de Neurocirugía, Hospital Juárez de México, Ciudad de México, México

\begin{abstract}
Resumen
Antecedentes: El Fenómeno de Kernohan-Woltman, o fenómeno de la escotadura de Kernohan, es un evento que revela un falso signo localizador de la lesión y que básicamente consiste en una hemiparesia por afección del pedúnculo cerebral desplazado hacia el lado contralateral y con dilatación pupilar del mismo lado. Objetivo: Puntualizar la dilatación pupilar como el signo localizador más confiable. Material y métodos: Se realizó una búsqueda en PubMed con la palabra clave "Kernohan". Resultados: La revisión de la literatura pone de manifiesto la confusión para localizar el sitio de la lesión previo a un estudio de imagen, siendo este imprescindible para la toma final de decisiones. Conclusiones: El fenómeno de Kernohan es una entidad que se caracteriza por dilatación pupilar ipsilateral a la lesión y una afectación del tracto corticoespinal contralateral que da manifestaciones clínicamente en el mismo lado de afectación de la pupila. La dilatación pupilar sigue siendo el signo localizador en este fenómeno, el cual debe siempre corroborarse por estudios de imagen.
\end{abstract}

Palabras clave: Kernohan. Escotadura. Fenómeno.

\begin{abstract}
Background: The Kernohan-Woltman Phenomenon or Kernohan Notch Phenomenon, is an event that reveals a false localizing sign of the lesion and that basically consists of hemiparesis due to involvement of the cerebral peduncle displaced to the contralateral side and with pupillary dilation of the same side. Objective: To point out pupillary dilation as the most reliable locator sign. Material and methods: A search was carried out in PUBMED with the keywords "Kernohan". Results: The literature review reveals the confusion to locate the site of the injury prior to an imaging study, being this essential for the final decision making. Conclusions: The Kernohan phenomenon is an entity characterized by pupillary dilation ipsilateral to the lesion and an affectation of the contralateral corticospinal tract that gives clinical manifestations on the same affected side of the pupil. Pupillary dilation remains the locator sign in this phenomenon, which must always be corroborated by imaging studies.
\end{abstract}

Key words: Kernohan. Notch. Phenomenon.

Correspondencia:

*Osvaldo M. San Martín-García

E-mail: neurosanmartin@gmail.com
Disponible en internet: 09-04-2021 Rev Hosp Jua Mex. 2021;88(1):37-43

www.revistahospitaljuarez.com 1405-9622/○ 2020 Sociedad Médico-Quirúrgica del Hospital Juárez de México, A.C. Publicado por Permanyer. Este es un artículo open access bajo la licencia CC BY-NC-ND (http://creativecommons.org/licenses/by-nc-nd/4.0/). 


\section{Introducción}

El síndrome de herniación uncal ocurre a consecuencia de una lesión intracraneal (de etiología traumática) que desplaza al lóbulo temporal hacia la línea causando una compresión del pedúnculo cerebral del mesencéfalo y de la porción subaracnoidea del nervio oculomotor del mismo lado".

La compresión de estas estructuras se manifiesta clínicamente como una dilatación pupilar y una oftalmoplejia ipsilateral a consecuencia de afectación del tercer nervio. El defecto motor del lado contrario, sea hemiparesia o hemiplejia, se explica debido al daño que sufren las fibras corticoespinales previo a su decusación a nivel del bulbo raquídeo.

Es importante señalar que este síndrome de herniación uncal viene acompañado de alteraciones del estado de alerta, componente característico del cuadro y que lo hace distinto del síndrome de Weber, en el cual se conserva el estado de consciencia y que además es de etiología vascular o neoplásica en algunas ocasiones.

La clínica neurológica que se observa en estos casos ocurre debido a la configuración interna del encéfalo y el daño que altera su armonía, pero a veces existe una variante que no cumple con la regla, una variante que presenta una dilatación pupilar que se acompaña con alteraciones motoras del mismo lado, lo que supone un reto para el clínico al momento de localizar el sitio exacto de afección y que debe tomar como referencia, pues se encuentra ante dos signos que parecen no ser del todo congruentes.

Esta variante en clínica neurológica se conoce como síndrome o fenómeno de la escotadura de Kernohan'.

El fenómeno de Kernohan-Woltman, o fenómeno de la escotadura de Kernohan, es un evento que revela un falso signo localizador de la lesión y que básicamente consiste en un defecto motor (hemiparesia o hemiplejia) por afectación del pedúnculo cerebral desplazado hacia el lado contralateral y con dilatación pupilar del mismo lado². Esto claramente dificulta el diagnóstico topográfico de la lesión.

Fue descrito por primera vez en 1927 por los alemanes Arnold Groeneveld y Georg Schaltenbrand, pero debido a la naturaleza de su trabajo y sus conductas amorales propias de la Alemania nazi de la época no recibieron el crédito por su labor y este fue otorgado a James Watson Kernohan en 1929, con una serie que contó en aquel entonces con 276 pacientes $^{3}$.

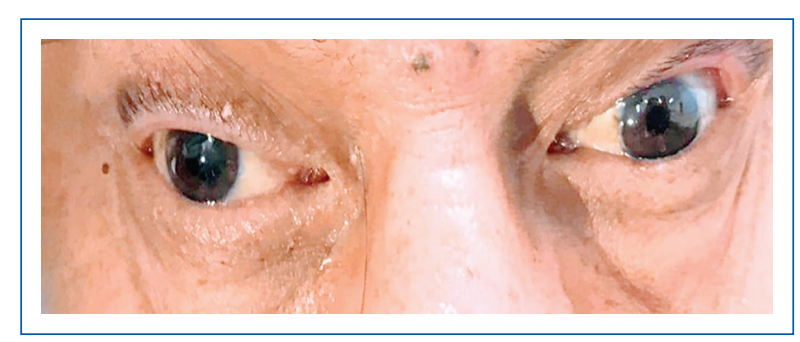

Figura 1. Midriasis derecha con afectación completa del tercer nervio craneal derecho.

\section{Material y métodos}

En el Servicio de Neurocirugía se estudió y trató a un paciente con antecedente de agresión con objeto contuso en la región cefálica derecha con posterior deterioro del estado de alerta rápidamente progresivo caracterizado por ausencia de repuesta verbal y motora. A su llegada al hospital se le encuentra estuporoso, con mirada primaria divergente a expensas del lado derecho, pupila derecha $5 \mathrm{~mm}$ sin respuesta luminosa refleja, pupila izquierda $3 \mathrm{~mm}$ arreactiva (Fig. 1), en el fondo de ojo derecho se observa edema de papila. El sistema motor tuvo extensión anormal frente a estímulo doloroso en hemicuerpo izquierdo con hemiplejia del lado derecho, hiperrreflexia generalizada con datos de liberación piramidal. Se realizó estudio tomográfico de encéfalo en cortes axiales desde la base hasta la convexidad observándose lesión ocupante de espacio que va de piso medio temporal a la convexidad hemisférica derecha, hiperdensa, compatible con hematoma subdural agudo además de hipodensidades dispersas compatibles con neumoencéfalo, desviación de la línea media que desplaza al mesencéfalo hacia el lado contralateral y que hace contacto con el borde tentorial, contusión hemorrágica frontal en polo y base de lóbulo temporal derecho (Fig. 2). Con los datos clínicos y radiológicos recabados se integró un síndrome de deterioro rostrocaudal en fase mesencefálica derivado de un fenómeno de Kernohan, se realizó intervención quirúrgica de urgencia para el drenaje de hematoma y posteriormente se vigiló al paciente en la unidad de cuidados intensivos.

Se realizó una revisión de la literatura sobre el fenómeno y a continuación hacemos una descripción detallada del sustrato anatomoclínico del padecimiento.

\section{Correlación anatomoclínica}

El fenómeno clásicamente está descrito que ocurre cuando una lesión (traumática) que incrementa 


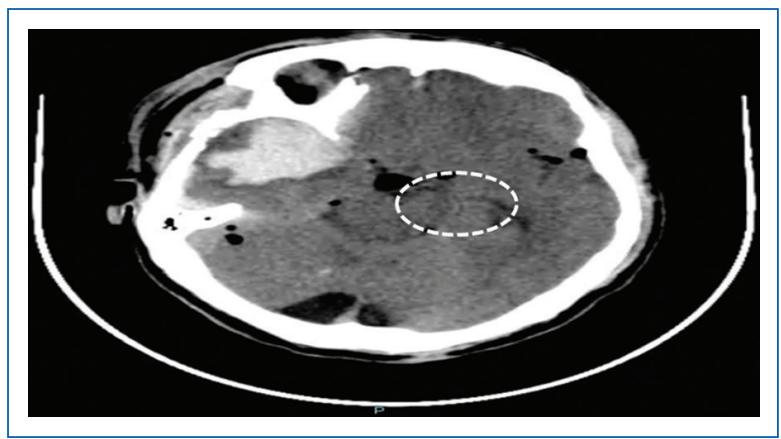

Figura 2. Tomografía computarizada de cráneo simple que muestra desplazamiento del mesencéfalo haciendo contacto con el borde libre del tentorio del otro lado.

progresivamente la presión intracraneal, causa un desplazamiento o basculación del tallo cerebral y consecuente compresión del pedúnculo cerebral sobre el borde libre del tentorio del lado contralateral, lo que provoca un daño a la vía corticoespinal previo a su decusación a nivel de la médula oblongada y se traduce en un déficit motor del mismo lado a la afectación pupilar $^{2,3}$.

En la clínica neurológica esta afección tiene dos variantes: el síndrome de herniación uncal propiamente dicho y el síndrome de la escotadura de Kernohan ${ }^{4}$. Hablando en concreto de este último, se caracteriza por afectación de las fibras del nervio oculomotor del mismo lado de la lesión, de las fibras corticoespinales del pedúnculo cerebral contralateral por basculación con el borde libre del tentorio y deterioro del estado de consciencia por interrupción de las fibras del sistema activador reticular ascendente (SARA).

\section{Consciencia}

El aumento súbito de la presión intracraneal condiciona un deterioro del estado de alerta al rebasar los mecanismos de compensación y autorregulación cerebral; generalmente se debe a eventos agudos y en este caso, a un traumatismo craneoencefálico severo. Las causas de un deterioro agudo de la consciencia se dividen en metabólicas y orgánicas; las metabólicas ocupan la gran mayoría de las causas, con un porcentaje aproximado del $80 \%$, seguido por un $20 \%$ en las orgánicas ${ }^{5}$, como es nuestro caso.

El compartimiento intracraneal no tiene la capacidad de expandirse cuando una masa ocupa un lugar en su interior, en este caso se trata de una lesión intracraneal rápidamente progresiva de origen traumático. Al incrementarse el volumen de la masa, el mecanismo de compensación inicia con el líquido cefalorraquídeo y continúa con la sangre en un periodo determinado de tiempo; finalmente pequeños aumentos de volumen rebasan estos mecanismos de compensación que se traducen en aumentos groseros de la presión intracraneal, proceso que clínicamente conocemos como síndrome de deterioro rostrocaudal ${ }^{1,2,5}$.

La evolución natural de la enfermedad dicta que el aumento de la presión comprime zonas centroencefálicas iniciando en diencéfalo hasta concluir en la parte más baja del tallo cerebral. Hablando del sitio de la lesión en este caso, el aumento de la presión también provoca cambios en el drenaje venoso de las venas perimesencefálicas, existe congestión y posteriormente hemorragias al no poderse rebasar la presión; estas hemorragias pueden afectar cualquier zona del mesencéfalo, entre ellas el tegmento.

Como se sabe, el SARA tiene su origen en mesencéfalo y puente alto en núcleos que se encargan de la producción de adrenalina (locus cerúleo), acetilcolina (núcleo pedúnculo pontino) y glutamato (núcleo reticular central pontino); la proyección de las fibras que parten de estos núcleos forma el haz tegmental central que llega hasta la sustancia gris periacueductal del mesencéfalo. Continuando su camino ascendente las fibras hacen otro relevo en los núcleos de la línea media del tálamo y este a su vez envía proyecciones a toda la corteza cerebral ${ }^{5}$.

Se considera que SARA es el encargado de mantener el estado de despierto y, en el caso de los seres humanos, de otorgar el estado de consciencia a través de vías que estimulan al cerebro y condicionan su respuesta ante estímulos de su medio externo ${ }^{1,5}$.

Lesiones estructurales de estos componentes provocan daños que se traducen clínicamente en alteraciones del estado de consciencia.

En la figura 3 se muestra un corte sagital del tallo cerebral en el que se señala la unión pontomesencefálica como el origen del SARA; obsérvese su estrecha relación con el acueducto de Silvio hasta su destino final en la sustancia gris periacueductal señalada en la figura 4. Esta última muestra un corte axial a nivel de los colículos superiores en donde es característica la presencia de los núcleos rojos, origen de la vía rubroespinal y cuya afectación se explica más adelante en este texto. En los pedúnculos cerebrales como zona de paso del tracto corticoespinal, este trayecto tiene una orientación longitudinal que posteriormente en el puente se encuentra en la zona intermedia entre las 


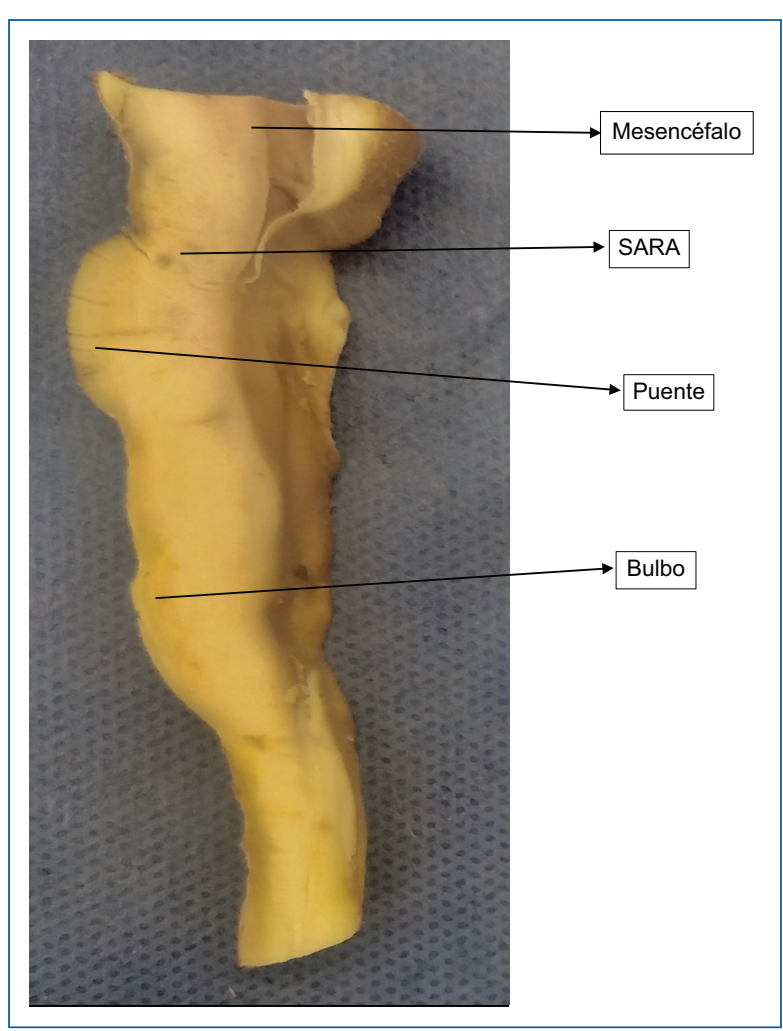

Figura 3. Vista lateral del tallo cerebral que muestra el origen del sistema activador reticular ascendente.

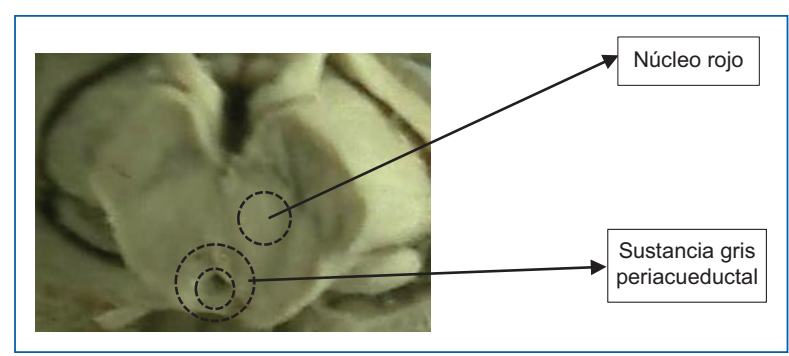

Figura 4. Corte transversal del mesencéfalo que muestra el destino del haz tegmental central hacia la sustancia gris periacueductal.

fibras transversales pontocerebelosas y las fibras corticonucleares.

\section{Oftalmología global derecha}

A la exploración neurológica se pudo apreciar una parálisis del tercer nervio craneal del lado derecho, observándose midriasis, ptosis y desviación de la mirada hacia afuera.

El origen real del nervio oculomotor se encuentra en un complejo nuclear bien definido y localizado a nivel superior del mesencéfalo en la región del tegmento 6 . La configuración funcional localiza en la periferia a las fibras parasimpáticas rodeando a las fibras eferentes somáticas generales; estas fibras parasimpáticas preganglionares tienen su origen en el núcleo parasimpático de Edinger-Westphal hasta alcanzar el ganglio ciliar; partiendo de aquí emergen las fibras posganglionares con información eferente visceral general hasta llegar a su destino en el esfínter de la pupila y el músculo ciliar ${ }^{6}$. Esto explica por qué el paciente inicialmente manifestó dilatación pupilar con posterior parálisis del ojo del mismo lado.

El nervio emerge del mesencéfalo de la fosa interpeduncular y recorre el borde medial del pedúnculo cerebral hasta alcanzar la pared lateral del seno cavernoso, recorre el borde superior de la misma pared y luego entra a la fisura orbitaria con los nervios IV, VI y porción oftálmica del V; recordar que una vez dentro de la cavidad orbitaria, el nervio atraviesa el anillo de Zinn y posteriormente se divide en una rama superior y otra inferior. Cada nervio posee de 8 a 16 raicillas individuales y recorre una distancia promedio de $24 \mathrm{~mm}$ hasta su porción cavernosa ${ }^{6}$. Por otro lado, el uncus sobrepasa el borde del tentorio aproximadamente de 3 a 4 $\mathrm{mm}$ y contacta directamente con el oculomotor en esta porción ${ }^{6}$, aunque hay algunas excepciones debido a variaciones anatómicas que se deben considerar.

Se sabe que el borde medial del oculomotor se encuentra separado de la apófisis clinoides posterior (previo a su entrada) $3 \mathrm{~mm}$ por debajo de esta, sin embargo, hay un pequeño porcentaje en donde la entrada del nervio es al mismo nivel o por detrás del borde lateral de la apófisis clinoides posterior ${ }^{6}$. Lo anterior traduce manifestaciones clínicas distintas cuando se trata de herniación uncal, sobre todo pupilares y que son de vital importancia al momento de realizar un diagnóstico topográfico.

La figura 4 muestra una vista inferior de un corte axial del mesencéfalo en el cual se observa la emergencia del nervio oculomotor entre los dos pedúnculos cerebrales y su recorrido hasta la fisura orbitaria superior además de su estrecha relación con el borde libre del tentorio, mostrado en la figura 5.

\section{Lesión de la vía corticoespinal}

Fue en 1870 cuando se dio un salto gigante en el campo de la neurología con el descubrimiento de que la corteza cerebral estaba organizada de tal manera que distintas zonas de la corteza motora primaria se proyectaban a distintas partes del cuerpo dando como 


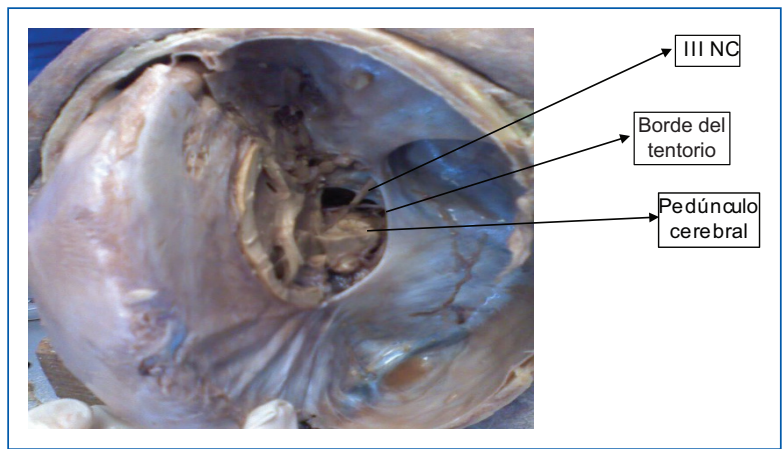

Figura 5. Vista superior del mesencéfalo.

NC: Nervio Craneal.

resultado los movimientos musculares voluntarios. Durante el siglo XX la estimulación eléctrica puso de manifiesto la correlación clínica contralateral de dichos movimientos y se identificaron zonas específicas en la zona que controlaban una $u$ otra parte de la musculatura?.

En nuestro caso el paciente presentó un cuadro de hemiplejia derecha con respuesta extensora anormal del lado izquierdo, es decir descerebración. Lo anterior se explica por una afectación tanto de la vía piramidal en su paso por el mesencéfalo como de las vías descendentes que se originan a partir del núcleo rojo.

Como se mencionó antes, el sistema de fibras de la corteza motora conduce los impulsos hasta el tallo cerebral y la médula espinal. Su origen se ubica en las fibras que proceden del área 4, del lóbulo paracentral, del área 6 y de los campos frontales de los ojos que corresponden al área 8 de Brodman; de la región poscentral parten fibras de las áreas 3,1 y 2 (área sensitiva primaria) y de las áreas 5 y 7 (área sensitiva secundaria). Es así como todas estas fibras en conjunto dan origen al tracto corticoespinal ${ }^{8-10}$.

Después de su origen, las fibras viajan en sentido caudal y atraviesan el brazo posterior de la cápsula interna hasta llegar al mesencéfalo en su porción peduncular; a este nivel es de particular interés el tegmento por la ubicación del SARA y los pedúnculos cerebrales propiamente dichos ${ }^{8}$.

La configuración del pedúnculo cerebral comprende la distribución de las fibras corticales que descienden desde su origen hasta su destino en la médula espinal y que se encuentran distribuidas en los tres quintos mediales que corresponden a la vía corticoespinal flanqueada a los lados en el quinto medial y quinto lateral por las fibras corticonucleares procedentes de la región frontal y temporal respectivamente ${ }^{8}$.

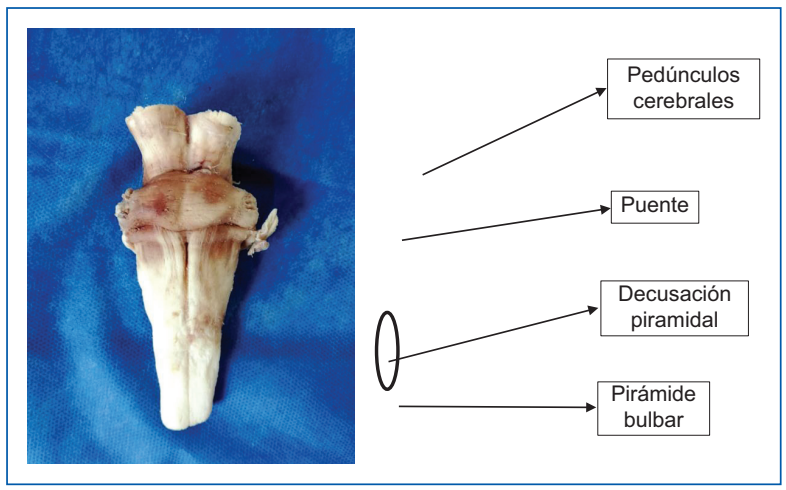

Figura 6. Vista anterior del tallo cerebral que muestra el recorrido de las fibras corticoespinales.

Después de pasar por el pedúnculo cerebral las fibras continúan su descenso por el puente en la porción basilar y una vez que se encuentran a nivel del bulbo raquídeo un gran porcentaje $(70-90 \%)$ se cruza al otro lado en la zona de transición con la médula espinal conocida como decusación piramidal o decapitación motora y descienden por el cordón lateral de la médula espinal; la figura 6 ilustra la descripción al presentar una vista anterior del tallo cerebral que muestra el recorrido de las fibras corticales en su paso por los distintos niveles del tallo cerebral antes de su entrecruzamiento, la zona contorneada representa la vía corticoespinal flanqueada por los tractos corticonucleares. El porcentaje restante de las fibras posterior a su decusación desciende en el cordón anterior de la médula espinal como haz corticoespinal anterior y también se cruza hacia el lado contrario, mientras unas pocas fibras permanecen del mismo lado en lo que se conoce como haz piramidal de Barnes ${ }^{11}$.

Dos tercios de las neuronas del tracto corticoespinal son de tipo mielinizadas ${ }^{12}$ y hacen conexiones con las neuronas motoras alfa de la médula espinal de las astas anteriores al mismo tiempo que también realizan conexiones con interneuronas y eventualmente también con neuronas motoras alfa y gama para otorgar las funciones de movilidad y tono muscular.

Partiendo de esta explicación, se entiende que tras una lesión que involucra al tracto corticoespinal se pierdan las capacidades motoras finas de las manos de manera irreversible quedando el resto de la extremidad unida en una sinergia flexora y/o extensora ${ }^{7}$. En el recorrido de la vía corticoespinal por todo el neuroeje es precisamente en el mesencéfalo donde se localiza el punto de afectación del síndrome o fenómeno de la escotadura de Kernohan. 
Tabla 1. Sustratos anatómicos del fenómeno de la escotadura de Kernohan

\begin{tabular}{|c|c|c|c|}
\hline Base neuroanatómica & Función & Manifestaciones clínicas & Topografía \\
\hline $\begin{array}{l}\text { Áreas corticales } \\
-\quad \text { Área } 4 \\
\text { - Área } 6 \\
\text { - Área } 8 \\
\text { - Áreas } 3,1 \text { y } 2 \\
\text { - Áreas } 5 \text { y } 7\end{array}$ & $\begin{array}{l}\text { Origen del tracto } \\
\text { corticoespinal Movimientos } \\
\text { voluntarios }\end{array}$ & $\begin{array}{ll}\text { - } & \text { Hemiparesia/ hemiplejia } \\
\text { - } & \text { Hiperreflexia } \\
\text { - } & \text { Hipertonía }\end{array}$ & \\
\hline Nervio oculomotor & $\begin{array}{l}\text { Inervación de la } \\
\text { musculatura extraocular, } \\
\text { músculo ciliar y la pupila }\end{array}$ & $\begin{array}{l}\text { - Midriasis } \\
\text { - Ptosis } \\
\text { - Desconjugación de la mirada }\end{array}$ & \\
\hline SARA & $\begin{array}{l}\text { Otorga el estado de } \\
\text { despierto y la consciencia }\end{array}$ & $\begin{array}{l}\text { Dificultad para mantener el estado de } \\
\text { despierto, incluso coma }\end{array}$ & \\
\hline Núcleo Rojo & $\begin{array}{l}\text { Origen del tracto } \\
\text { rubroespinal } \\
\text { Flexión miembros } \\
\text { superiores }\end{array}$ & Extensión anormal de descerebración & \\
\hline
\end{tabular}

\section{Respuesta extensora anormal}

En el mesencéfalo se encuentra el origen de la vía rubroespinal a partir del núcleo rojo (Fig. 4) que se encarga de otorgar la función flexora de los miembros superiores. Localizado en el mesencéfalo alto en la porción tegmental, el núcleo rojo da origen a un grupo de fibras que se decusan por delante en la llamada decusación de Forel, de donde parten las vías rubroespinales que llegan al cordón lateral y al asta anterior en la médula espinal, para de ahí distribuirse somatotópicamente en la musculatura flexora de los brazos.

Al presentarse el deterioro rostrocaudal que afecta estas estructuras se pierde la función del haz rubroespinal (flexión-descorticación), dando pie a que predomine la actividad de la vía vestibuloespinal, cuyo papel comprende la inervación de la musculatura extensora axial y apendicular (descerebración). Lo anterior se explica porque los núcleos vestibulares se encuentran en una porción más caudal en relación con el mesencéfalo, es decir puente y bulbo raquídeo, por lo que la actividad de esta vía se encuentra $100 \%$ conservada y predomina sobre la dañada, en este caso la vía rubroespinal.

Es así que la respuesta extensora anormal localiza a la lesión en mesencéfalo y se corresponde con el resto de signos clínicos hallados en el síndrome de la escotadura de Kernohan.

\section{Material y métodos}

Se realizó exploración neurológica a un paciente con antecedente de traumatismo craneoencefálico cuyos hallazgos topográficos eran incongruentes con la clínica que presentaba; se realizó estudio complementario de tomografía computarizada de cráneo, que mostró los hallazgos previamente descritos para tan inusual variante.

\section{Resultados}

El resultado fue una variante del síndrome de deterioro rostrocaudal conocida como fenómeno de la escotadura de Kernohan, cuya resolución se llevó a cabo mediante drenaje quirúrgico de la lesión 
intracraneal. Las figuras 2, 5 y 6 muestran la correlación neuroanatómica que explica los hallazgos clínicos típicos de esta variante, así como el papel crucial que juegan los estudios de imagen.

\section{Discusión}

Nuestros hallazgos clínicos y radiológicos coinciden con lo reportado en la literatura, en cuyos primeros casos reportados se tomó como signo localizador a la afección pupilar para la toma de decisiones, pues se debe recordar que en aquel entonces no se contaba con estudios de imagen.

\section{Conclusión}

El fenómeno de Kernohan es una entidad que se caracteriza por dilatación pupilar ipsilateral a la lesión y una afectación del tracto corticoespinal contralateral que da manifestaciones clínicamente en el mismo lado de afección de la pupila. La dilatación pupilar sigue siendo el signo localizador en este fenómeno, el cual debe siempre corroborarse por estudios de imagen.

La tabla 1 muestra un resumen de las estructuras anatómicas involucradas en el fenómeno de la escotadura de Kernohan, su función y las manifestaciones clínicas por lesión.

\section{Agradecimientos}

Expresamos nuestro agradecimiento al Dr. Rafael Mendizábal Guerra y al Dr. Julio César Soto Barraza por facilitar el entendimiento del sustrato neuroanatómico y recordarnos siempre la importancia de la clínica neurológica.

\section{Financiamiento}

Los autores no recibieron patrocinio para llevar a cabo este artículo.

\section{Conflicto de intereses}

Los autores declaran no tener conflicto de intereses alguno.

\section{Responsabilidades éticas}

Protección de personas y animales. Los autores declaran que para esta investigación no se han realizado experimentos en seres humanos ni en animales.

Confidencialidad de los datos. Los autores declaran que han seguido los protocolos de su centro de trabajo sobre la publicación de datos de pacientes.

Derecho a la privacidad y consentimiento informado. Los autores han obtenido el consentimiento informado de los pacientes y/o sujetos referidos en el artículo. Este documento obra en poder del autor de correspondencia.

\section{Bibliografía}

1. Carrillo Esper R, Guinto Balanzar G, Castelazo Arredondo JA. Traumatismo craneoencefálico. México, D.F.: Editorial Alfil; 2010

2. Zhang $\mathrm{CH}$, DeSouza RM, Kho JSB, Vundavalli S, Critchley G. Kernohan-Woltman notch phenomenon: a review article. $\mathrm{Br} \mathrm{J}$ Neurosurg. 2017;31(2):159-66.

3. Juan DF. Fenómeno de la escotadura de Kernohan. Neurocienc Colomb. 2017;24(1).

4. Haines DE. Neuroanatomía clínica: Texto y atlas. Lippincott Williams \& Wilkins; 2015

5. Posner JB, Saper CB, Schiff ND, Claassen J. Plum and Posner's diagnosis of stupor and coma. 5. ${ }^{\mathrm{a}}$ ed. Oxford University Press; 2008.

6. Cruz HR. Nervios craneanos y algunas consideraciones clínicas. Litográfica Century; 1984.

7. Kandel ER. Principios de neurociencia. 4. ${ }^{\mathrm{a}}$ ed. McGraw-Hill Interamericana; 2000.

8. Segura JN. Neuroanatomía funcional: Síndromes neurológicos. Impresiones Modernas; 1976.

9. Paxinos G, Mai JK. Front Matter. En: Paxinos G, Mai JK, editores. The human nervous system. Second edition. San Diego: Academic Press; 2004.

10. Nieuwenhuys $R$, Voogd J, van Huijzen $C$. The human central nervous system. Schweiz Arch Für Neurol Psychiatr. 2009;160(02):82.

11. Antúnez LL. Anatomía funcional del sistema nervioso. Limusa; 1979.

12. Carpenter MB, Kaufman A. Neuroanatomía: fundamentos. Panamericana; 1994. 